\title{
Integrable Equations and Their Evolutions Based on Intrinsic Geometry of Riemann Spaces
}

\author{
Paul Bracken \\ Department of Mathematics, University of Texas, Edinburg, TX 78541-2999, USA \\ Correspondence should be addressed to Paul Bracken, bracken@panam.edu \\ Received 9 March 2009; Accepted 11 August 2009 \\ Recommended by Peter Basarab-Horwath
}

The intrinsic geometry of surfaces and Riemannian spaces will be investigated. It is shown that many nonlinear partial differential equations with physical applications and soliton solutions can be determined from the components of the relevant metric for the space. The manifolds of interest are surfaces and higher-dimensional Riemannian spaces. Methods for specifying integrable evolutions of surfaces by means of these equations will also be presented.

Copyright (C) 2009 Paul Bracken. This is an open access article distributed under the Creative Commons Attribution License, which permits unrestricted use, distribution, and reproduction in any medium, provided the original work is properly cited.

\section{Introduction}

Surfaces arise in the study of many classical systems, especially those in which interfaces or fronts appear. This occurs in the study of surface waves, deformations of membranes, and boundaries between regions of differing viscosity or densities. Surfaces are also finding applications at present in the areas of classical string theory and quantum field theory $[1,2]$. Two cases which are particularly important for applications and have received much attention are the cases in which the surface can be ascribed a constant mean curvature or a constant Gaussian curvature. Each of these cases has a wide variety of nonlinear partial differential equations associated with it, and each has been studied with great interest recently $[3,4]$.

This is due to the fact that many partial differential equations, or systems of differential equations, can be generated as a consequence of formulas which result from the study of surfaces. Some equations such as those related to constant mean curvature are a consequence of the theory, and many related to constant Gaussian curvature can be obtained as a result of specifying quantities which are in the formulas in a particular form. Moreover, many of these equations are known to possess soliton solutions [5], and so this provides a link between equations, solitons, and surfaces. This has certainly been shown to be the case in the study of constant mean curvature surfaces [6]. In fact, many nonlinear equations which have been 
studied recently, such as the $\mathrm{KdV}$ and $\mathrm{mKdV}$ equations, are directly related to surfaces which have constant Gaussian curvature $[7,8]$. These equations have soliton solutions, and conversely solutions from these equations can be used to determine the first and second fundamental forms of a surface. Historically, Bianchi and Bäcklund introduced symmetry transformations which can be regarded as transformations between surfaces and have come to be called Bäcklund transformations. This was initially done for the sine-Gordon equation which allows the construction of new pseudospherical surfaces from a given surface. In an approach that uses the inverse scattering transform method, one starts with the system of $1+1$-dimensional linear problems $\psi_{x}=P \psi, \psi_{y}=Q \psi$ and then constructs explicit formulas for the immersion of one-parameter families of surfaces [9].

The study of the general theory of three-dimensional Riemann spaces has not been completed as far as a theory of surfaces is concerned [10]. In fact, various partial differential equations do arise within the study of problems in three and higher dimensional Riemann spaces as will be seen here. Three-dimensional Riemann spaces and possibly even higher dimensional versions play a crucial and fundamental role in general relativity and the study of gravity in general. One of the reasons for this is that any metric in general relativity can be transformed into a synchronic system of coordinates, which is one in which the spatial part of the metric is Riemannian. In this event, an initial value problem can be formulated in terms of three-dimensional Riemannian space plus one time variable in a very natural way. The study of higher-dimensional Riemann spaces can very well be of importance in the study of Kaluza-Klein theories as well as in formulating string theories.

The intrinsic geometry of surfaces and Riemannian spaces will be introduced and described without any reference to the enveloping space. Results from the study of these spaces are used to obtain nonlinear differential equations. This gives an automatic connection between surfaces and equations. The procedure will be to define a metric in mathematical form with a particular structure and a curvature which will depend on the components of the metric and given by a specific formula, in particular the curvature equation. This will be used to establish a relationship between the intrinsic geometry of the spaces as specified by the functions in the metric and multidimensional integrable equations which result from the curvature equation [11]. Methods for specifying integrable evolutions or deformations of surfaces will also be examined as well. The study of the solutions to these equations is not the main objective here, but to formulate some new, relevant equations and propose some new ones for study.

\section{Nonlinear Equations Related to Surfaces}

Consider two-dimensional surfaces which have a first fundamental form given in terms of components as

$$
d s_{I}^{2}=E d x^{2}+2 F d x d y+G d y^{2}
$$

where $E, F$, and $G$ are functions of the local coordinates $(x, y)$ of a surface. The basic invariant which is characteristic of the intrinsic geometry of a surface is the Gaussian curvature, which is determined by

$$
K=\frac{R_{1212}}{g}=\frac{R_{1212}}{H^{2}}
$$


In (2.2), $R_{1212}$ is the nonzero component of the Riemann tensor $R_{\alpha \beta \gamma \delta}$, and $H$ is related to $g$ by means of the expression [12]

$$
g=E G-F^{2}=H^{2}
$$

Given the components of the first fundamental form or metric, the Christoffel symbols $\Gamma_{i j}^{k}$ are evaluated by means of

$$
\Gamma_{i j}^{k}=\frac{1}{2} g^{k s}\left(\frac{\partial g_{i s}}{\partial x^{j}}+\frac{\partial g_{j s}}{\partial x^{i}}-\frac{\partial g_{i j}}{\partial x^{s}}\right) .
$$

The component $R_{1212}$ can be calculated by means of

$$
R_{1212}=\frac{\partial \Gamma_{122}}{\partial x}-\frac{\partial \Gamma_{121}}{\partial y}+\Gamma_{11}^{h} \Gamma_{2 h 2}-\Gamma_{12}^{h} \Gamma_{2 h 1}
$$

In terms of the components of the metric of a surface given by (2.1), the Gaussian curvature $K(x, y)$ can be evaluated by first calculating (2.5) and then putting this result into (2.2). This procedure shows that the Gaussian curvature $K(x, y)$ is related to the components of the metric of the surface by means of the Gauss equation

$$
K=\frac{1}{2 H}\left(\left(\frac{F}{E H} E_{y}-\frac{1}{H} G_{x}\right)_{x}+\left(\frac{2}{H} F_{x}-\frac{1}{H} E_{y}-\frac{F}{E H} E_{x}\right)_{y}\right)
$$

By specifying the components of the metric (2.1) in a particular way, it is possible to use (2.6) to generate various types of nonlinear equations. Several examples will serve to illustrate the main idea as to exactly how this can be done.

First put $E=G=0$ and $H=F$ in (2.6), then we obtain

$$
(\ln F)_{x y}-F K=0 .
$$

Setting $F=\exp (\varphi)$, this equation becomes

$$
\varphi_{x y}-K \exp (\varphi)=0
$$

The coordinate curves are minimal lines, and for the case of constant Gaussian curvature $K$, it specifies a particular nonlinear equation, the Liouville equation.

As another example, suppose that we take $F=0, E=\cos ^{2} \theta$, and $G=\sin ^{2} \theta$, then $H=\cos \theta \sin \theta, G_{x}=2 \sin \theta \cos \theta \theta_{x}$, and $E_{y}=-2 \cos \theta \sin \theta \theta_{y}$, and one has the lines of curvature as the coordinate curves. The Gauss equation (2.6) takes the form

$$
\theta_{x x}-\theta_{y y}=-H K
$$


Taking a constant negative curvature $K=-2 a^{2}$ in (2.9), it is found that $\theta$ satisfies

$$
\theta_{x x}-\theta_{y y}=a^{2} \sin (2 \theta)
$$

Thus, for different metrics, or systems of coordinates, on a surface, the Gauss equation (2.6) reduces to a simple partial differential equation, as in (2.8) or (2.10). These equations have come up within the analysis of the pure geometry of surfaces. The well-known cases of nonlinear partial differential equations, such as the Liouville and sine-Gordon equations, correspond to the case of constant Gaussian curvature.

Finally, another equation which has many applications to physics can be developed in this way. Consider a geodesic system of coordinates for which $E=1, F=0$, and (2.1) reduces to the diagonal form $d s^{2}=d x^{2}+G d y^{2}$. Thus the curves $y$ equal constant are geodesics, and for (2.3) in this metric, this implies that $G=H^{2}$. The Gauss equation takes the form

$$
H_{x x}+K(x, y) H=0 .
$$

This is a linear equation, and when $K$ is fixed to be constant, the corresponding surfaces are the spherical or pseudospherical surfaces of constant curvature. It is important to realize that (2.11) is closely connected with the one-dimensional stationary Schrödinger equation $[13,14]$. This can be seen by identifying

$$
H(x, y)=\operatorname{Re} \psi(x, y, \lambda), \quad K(x, y)=-U(x, y)+\lambda_{0}^{2},
$$

where Re denotes the real part, and $\lambda_{0}$ is an arbitrary real or pure imaginary value of the spectral parameter $\lambda$ in the equation

$$
-\psi_{x x}+U(x, y) \psi=\lambda^{2} \psi
$$

Therefore, solvable cases of the Schrödinger equation (2.13) provide the explicit expressions for the Gauss curvature $K(x, y)$ and the metric of surfaces referred to their geodesic coordinates. Here in (2.13), the quantity $y$ acts as a parameter. In fact, in general, one can prescribe any dependence of $K(x, y)$ and $H(x, y)$ on the variable $y$.

A way in which the dependence on $y$ can be fixed is to require that the function $H$ obeys the additional linear equation

$$
H_{y}=A\left(K, K_{x}, \partial_{x}\right) H
$$

where $A$ is some additional differential operator. Recalling (2.12), where $\psi$ obeys (2.13), we require that in addition to (2.13), the function $\psi$ satisfies an equation of the form

$$
\psi_{y}=A\left(U, U_{x}, \ldots, \partial_{x}\right) \psi,
$$

where $A$ is the linear differential operator in $\partial_{x}$. The compatibility of (2.13) and (2.15) gives the preservation of (2.11) in the $y$ variable. Moreover, the compatibility condition for 
(2.13) and (2.15) is equivalent to a nonlinear partial differential equation for $U$. As a simple example, take $A=c \partial_{x}$, where $c$ is a constant, then $\psi$ satisfies the two equations

$$
\psi_{x x}=\left(U-\lambda^{2}\right) \psi, \quad \psi_{y}=c \partial_{x} \psi
$$

Differentiating the second equation twice with respect to $x$ yields

$$
\partial_{x}^{2} \partial_{y} \psi=c\left(\partial_{x} U\right) \psi+c U \partial_{x} \psi-c \lambda^{2} \partial_{x} \psi
$$

Similarly, differentiating the first equation with respect to $y$ gives

$$
\partial_{y} \partial_{x}^{2} \psi=\left(\partial_{y} U\right) \psi+c U \partial_{x} \psi-c \lambda^{2} \partial_{x} \psi
$$

Equating these two derivatives and simplifying the result, a first-order equation for $U$ is obtained, namely, $c \partial_{x} U-\partial_{y} U=0$. The general solution to this equation is $U=U(x+c y)$ and hence $K=K(x+c y)$. In fact, a nonlinear equation arises with the choice of $A$ as a third-order differential operator.

Theorem 2.1. Suppose (2.15) is given by

$$
4 \psi_{x x x}-6 U \psi_{x}-3 U_{x} \psi+\psi_{y}=0 .
$$

Then the compatibility condition for (2.13) and (2.19) is equivalent to the following equation for $U$ :

$$
U_{x x x}-6 U U_{x}+U_{y}=0
$$

Proof. Substituting (2.13) into (2.19), we have the pair

$$
\psi_{x x}=(U-\lambda) \psi, \quad \psi_{y}=-U_{x} \psi+2 U \psi_{x}+4 \lambda^{2} \psi_{x} .
$$

Calculating the compatibility condition $\psi_{x x y}-\psi_{y x x}=0$ based on (2.21), in order to enforce this, it is found that (2.20) must hold. Putting $K(x, y)=-U(x, y)+\lambda_{0}^{2},(2.20)$ takes the form

$$
K_{x x x}+6 K K_{x}-6 \lambda_{0}^{2} K_{x}+K_{y}=0 .
$$

Equations (2.20) and (2.22) are precisely the KdV equation, which is known to be integrable. The basic idea of the method is to generate a solvable nonlinear system from the compatibility condition of linear partial differential equations with variable coefficients. Of course, the KdV equation has exact solutions, and so surfaces may be generated with soliton curvature and associated solitonic metric. Therefore, as an example, it can be checked that $U$ given below is a solution to (2.20):

$$
U(x, y)=\frac{-2 a^{2}}{\cosh ^{2}\left(a\left(x-4 a^{2} y\right)-x_{0}\right)}, \quad K(x, y)=\frac{2 a^{2}}{\cosh ^{2}\left(a\left(x-4 a^{2} y\right)-x_{0}\right)}+\lambda_{0}^{2} .
$$


The coefficient $H(x, y)$ in the metric obeys a nonlinear equation such as (2.22) for $K$. This can be obtained by solving (2.13) for $U$ and substituting (2.19) to give

$$
\psi_{x x x}-6 \lambda^{2} \psi_{x}-3 \frac{\psi_{x} \psi_{x x}}{\psi}+\psi_{y}=0
$$

\section{Evolutions of Surfaces}

Consider now integrable evolutions or deformations of surfaces which are referred to geodesic coordinates [15]. Suppose that the coefficient $G$ or $H$ of the metric and the Gaussian curvature depend on an additional parameter which can be thought of as a time variable; so we write $H=H(x, y, t), K=K(x, y, t)$. Evolutions of $H$ and $K$ in $t$ can be sought which preserve (2.11), or by virtue of (2.12), evolutions in $t$ which preserve (2.13). They are given by the compatibility condition of (2.13) and a linear equation of the form (2.15) with $y$ replaced by $t$; so there exists the pair

$$
-\psi_{x x}+U(x, t) \psi=\lambda^{2} \psi, \quad \psi_{x}=A\left(U, U_{x}, \ldots, \partial_{x}\right) \psi
$$

where $A$ is a linear differential operator. The evolutions which can be taken for $A$ can come from the KdV hierarchy [16, 17].

Dynamics for a surface can also be introduced by fixing the dependence of $K$ and $H$ using one of the equations from the KdV hierarchy and then specifying the evolution in $t$ by another equation from the hierarchy. The common solution $K(x, y, t)$ of the $\mathrm{KdV}$ equation (2.22) and a higher $\mathrm{KdV}$ equation, for example, $K_{t}+\partial_{x}^{2 n+1} K+\cdots=0$ generates the evolution of surfaces.

Another way for fixing the dependence of $K$ and $H$ on $y$ and determination of the evolution in $t$ consists in considering the compatibility of a system composed of (2.13) and an equation of the form

$$
\psi_{t}+f\left(\partial_{x}^{2}+K\right) \psi_{y}+\sum_{n=1}^{M} U_{n}(x, y, t) \partial_{x}^{n} \psi=0
$$

where $f$ is an arbitrary polynomial, and $U_{n}$ are functions. The compatibility of (2.13) and (3.2) is equivalent to a 2+1-dimensional integrable equation for $K$. The following theorem gives a case in point.

Theorem 3.1. (i) Consider the following pair of evolution equations with $U=-K$ :

$$
\begin{gathered}
\psi_{x x}=-K(x, y, t) \psi, \\
\psi_{t}=-2\left(\partial_{x}^{2} \psi_{y}+K \psi_{y}\right)-2\left[\int_{-\infty}^{x} d s K_{y}(s, y, t)\right] \psi_{x}-K_{y} \psi,
\end{gathered}
$$


where here and in what follows one defines

$$
\partial_{x}^{-1} \kappa(x, y)=\int_{-\infty}^{x} d s \kappa(s, y)
$$

Then the compatibility condition of (3.3) and (3.4) is equivalent to the following equation for $K(x, y, t)$ :

$$
K_{t}+K_{x y y}+4 K K_{y}+2 K_{x} \partial_{x}^{-1} K_{y}=0 .
$$

(ii) Consider the following pair of evolution equations:

$$
\begin{gathered}
\psi_{x x}=-K(x, y, t) \psi+\lambda^{2} \psi, \\
\psi_{t}=-2\left(\partial_{x}^{2} \psi_{y}+K \psi_{y}\right)-2\left(\int_{-\infty}^{x} d s K_{y}(s, y, t)\right) \psi_{x}-K_{y} \psi .
\end{gathered}
$$

The compatibility condition for these two equations implies that $K$ satisfies

$$
K_{t}+K_{x x y}+4 K K_{y}+2 K_{x} \partial_{x}^{-1} K_{y}-2 \lambda^{2} K_{y}=0
$$

The proof of this Theorem runs along exactly the same lines as Theorem 2.1. All of the lengthy calculations throughout have been done with Maple [18].

Equation (3.6), for example, has an infinite set of integrals of motion, which are

$$
C_{n}=\iint d x d y P_{n}(x, y)
$$

where the densities $P_{n}$ are given by the recursion

$$
P_{m+1}=P_{m, x}+\sum_{k=1}^{m-1} P_{k} P_{m-k}, \quad P_{1}=-K(x, y, t), \quad m=1,2,3, \ldots
$$

The first such integrals of motion turn out to be

$$
C_{1}=\iint d x d y K(x, y, t), \quad C_{2}=\iint d x d y K^{2}(x, y, t), \quad C_{3}=\iint d x d y\left(K_{x}^{2}-2 K^{3}\right) .
$$

Thus, there exists an infinite set of global characteristics of a surface that are invariant under the evolution discussed in Theorem 3.1.

As a generalization of (3.3) and (3.7) in Theorem 3.1, we have the theorem which follows. 
Theorem 3.2. Consider the following pair of evolution equations:

$$
\begin{gathered}
\partial_{x}^{2} \psi+\sigma \partial_{y} \psi+K(x, y, t) \psi=0 \\
\partial_{t} \psi+4 \partial_{x}^{3} \psi+6 K \partial_{x} \psi+3 K_{x} \psi-3 \sigma\left(\partial_{x}^{-1} K_{y}\right) \psi+\alpha=0
\end{gathered}
$$

where $\sigma^{2}= \pm 1$ and $\alpha$ is a constant. The compatibility condition for these two equations implies that $K$ satisfies

$$
K_{t x}+K_{x x x x}+6\left(K K_{x}\right)_{x}+3 \sigma^{2} K_{y y}=0
$$

This 2+1-dimensional equation is integrable.

A more systematic method [19] for passing to higher dimensions involves considering the compatibility condition for a pair of equations of the form

$$
\begin{gathered}
L \psi=\lambda \psi, \\
\sum_{k} f_{k}(L) \frac{\partial \psi}{\partial z_{k}}+A \psi=0,
\end{gathered}
$$

where $f_{k}$ are arbitrary polynomials in the operator $L$, and $z_{k}$ are the variables. A slightly different reformulation of this system will be of particular interest here, namely,

$$
\begin{gathered}
L \psi=\lambda \psi, \\
(D+A) \psi=\sum_{k} \lambda^{k} \frac{\partial \psi}{\partial z_{k}}+A \psi=0 .
\end{gathered}
$$

In particular, if there are three variables and we take $D \psi=\left(\lambda \partial_{y}+\partial_{t}\right) \psi$, with $L \psi=\left(\partial_{x}^{2}+K\right) \psi$, then (3.16) takes the form

$$
\partial_{x}^{2} \psi=-K \psi+\lambda \psi, \quad \partial_{t} \psi=-\lambda \partial_{y} \psi-A \psi
$$

Differentiating the first of the equations in (3.17) with respect to $t$ and the second with respect to $x$ twice, the compatibility condition for (3.17) takes the following form:

$$
\partial_{t}(L-K) \psi+\lambda \partial_{y}(L-K) \psi+(L-K) A \psi=0
$$

Expanding this out, we have the explicit form

$$
-K_{t} \psi+K\left(\lambda \partial_{y} \psi+A \psi\right)-\lambda\left(\lambda \partial_{y} \psi+A \psi\right)+\lambda \partial_{y}(-K \psi+\lambda \psi)+\partial_{x}^{2}(A \psi)=0
$$


Let us work out (3.19) explicitly for the case in which $A$ is the operator

$$
A=\alpha \partial_{x}^{3}+f \partial_{x}+g
$$

where $\alpha$ is an arbitrary constant, and $f$ and $g$ depend on $x$.

Theorem 3.3. With operator A given by (3.20), (3.19) is given explicitly as follows:

$$
\begin{aligned}
\left(K_{t}+\right. & \left.\alpha K_{x x x}+(-3 \alpha K+f+3 \alpha \lambda) K_{x}+\lambda K_{y}-2 \lambda f_{x}-g_{x x}+2 f_{x} K\right) \psi \\
& +\left(3 \alpha K_{x x}-f_{x x}-2 g_{x}\right) \psi_{x}=0 .
\end{aligned}
$$

The last term in $\psi_{x}$ is absent from (3.21) provided that $f$ and $g$ satisfy the equation

$$
f_{x x}+2 g_{x}-3 \alpha K_{x x}=0
$$

Therefore when (3.22) is satisfied, compatibility condition (3.19) holds if the equation for $K$ multiplying $\psi$ in (3.21) vanishes, namely,

$$
K_{t}+\alpha K_{x x x}+(-3 \alpha K+f+3 \alpha \lambda) K_{x}+\lambda K_{y}-2 \lambda f_{x}-g_{x x}+2 f_{x} K=0 .
$$

To prove Theorem 3.3, the compatibility condition for (3.17) is worked out, and the higher derivatives of $\psi$ in $x$ are eliminated using the first equation of (3.17). This is a long calculation.

A solution to (3.22) can be determined. Let us set

$$
f=\tilde{f}+\int_{-\infty}^{x} K_{y}(s, y, t) d s, \quad g=c\left(K_{y}+\alpha K_{x}\right),
$$

where constant $c$ will be determined in the process. Substituting (3.24) into (3.22), the solution will work if $c=-1 / 2$ and $\tilde{f}$ is determined from

$$
\tilde{f}_{x x}=4 \alpha K_{x x}
$$

which implies that we can take $\tilde{f}=4 \alpha K$. Let us summarize these results in the following theorem.

Theorem 3.4. Let $A$ be given by (3.20) with $f$ and $g$ given by

$$
f=4 \alpha K+\int_{-\infty}^{x} K_{y} d s, \quad g=-\frac{1}{2}\left(K_{y}+\alpha K_{x}\right) .
$$

Then (3.19) is satisfied identically provided that $K$ satisfies the partial differential equation

$$
K_{t}+\frac{1}{2}\left(3 \alpha K_{x x x}+K_{x x y}\right)+\left(\int_{-\infty}^{x} K_{y} d s+9 \alpha K-5 \alpha \lambda\right) K_{x}+(2 K-\lambda) K_{y}=0 .
$$




\section{Scalar Curvature of Three and Four-Dimensional Riemann Spaces}

In the previous section, it has been seen that the Gauss equation for the two-dimensional Riemann spaces connects the Gaussian curvature $K$ to the coefficients of the metric. In fact, two of the three components of the surface metric can always be transformed away by a change of variables.

For three-dimensional Riemann spaces, by means of a transformation of coordinates, the corresponding metric can be converted to diagonal form

$$
d s^{2}=e_{1} H_{1}^{2} d x_{1}^{2}+e_{2} H_{2}^{2} d x_{2}^{2}+e_{3} H_{3}^{2} d x_{3}^{2}
$$

where $e_{i}= \pm 1$ allow us to control the signs in the metric and $H_{i}$ are functions. In terms of the components of the metric, the scalar curvature is given by

$$
R=\sum g^{i l} g^{k m} R_{i k l m}
$$

For the diagonal metric (4.1), $R$ can be evaluated explicitly, and the scalar curvature takes the following form:

$$
\begin{aligned}
-\frac{1}{2} R= & \frac{1}{H_{1} H_{2}}\left[e_{2} \frac{\partial}{\partial x_{2}}\left(\frac{1}{H_{2}} \frac{\partial H_{1}}{\partial x_{2}}\right)+e_{1} \frac{\partial}{\partial x_{1}}\left(\frac{1}{H_{1}} \frac{\partial H_{2}}{\partial x_{1}}\right)\right] \\
& +\frac{1}{H_{1} H_{3}}\left[e_{1} \frac{\partial}{\partial x_{1}}\left(\frac{1}{H_{1}} \frac{\partial H_{3}}{\partial x_{1}}\right)+e_{3} \frac{\partial}{\partial x_{3}}\left(\frac{1}{H_{3}} \frac{\partial H_{1}}{\partial x_{3}}\right)\right] \\
& +\frac{1}{H_{2} H_{3}}\left[e_{2} \frac{\partial}{\partial x_{2}}\left(\frac{1}{H_{2}} \frac{\partial H_{3}}{\partial x_{2}}\right)+e_{3} \frac{\partial}{\partial x_{3}}\left(\frac{1}{H_{3}} \frac{\partial H_{2}}{\partial x_{3}}\right)\right] \\
& +\frac{1}{H_{1} H_{2} H_{3}}\left[\frac{e_{1}}{H_{1}} \frac{\partial H_{2}}{\partial x_{1}} \frac{\partial H_{3}}{\partial x_{1}}+\frac{e_{2}}{H_{2}} \frac{\partial H_{1}}{\partial x_{2}} \frac{\partial H_{3}}{\partial x_{2}}+\frac{e_{3}}{H_{3}} \frac{\partial H_{1}}{\partial x_{3}} \frac{\partial H_{2}}{\partial x_{3}}\right] .
\end{aligned}
$$

The factor $-1 / 2$ on the left side arises from a factor of -2 on the right-hand side divided out.

Let us begin with the simplest case for which $H_{1}=H_{2}=1$ and $H_{3}=H(x, y, z)$ with $e_{1}=e_{3}=1, e_{2}=\sigma^{2}$. In this case, (4.3) takes the form

$$
H_{x x} \pm H_{y y}+\frac{1}{2} R H=0
$$

By setting

$$
-\frac{1}{2} R=U-E, \quad H=\psi,
$$

(4.4) can be put in the equivalent form

$$
-\left(\partial_{x}^{2}+\sigma^{2} \partial_{y}^{2}\right) \psi+U(x, y, z) \psi=E \psi
$$


For the case $\sigma^{2}=1,(4.6)$ becomes the two-dimensional stationary Schrödinger equation, while for the case $\sigma^{2}=-1$ the perturbed string equation results.

As in the two-dimensional case, one can specify the dependence of the scalar curvature $R$ on another variable $z$ by requiring that $H$, and hence $\psi$, obeys an additional linear partial differential equation compatible with (4.6). Consider Riemann spaces such that the scalar curvature and metric obey the linear system of equations

$$
-\left(\partial_{x}^{2}+\sigma^{2} \partial_{y}^{2}\right) H-\frac{1}{2} R H=0, \quad H_{z}+\tilde{A}\left(R, \ldots, \partial_{x}, \partial_{y}\right) H=0 .
$$

In (4.7), $\widetilde{A}$ is a linear partial differential operator. Equivalently, in terms of $U$ and $V$, the equations in (4.7) can be written as

$$
-\left(\partial_{x}^{2}-\sigma^{2} \partial_{y}^{2}\right) \psi+U \psi=E \psi, \quad \psi_{z}+\tilde{A}\left(U, \ldots, \partial_{x}, \partial_{y}\right) \psi=0
$$

The compatibility condition for this system is equivalent to a nonlinear partial differential equation for the function $U$. It will be useful to write the first equation in (4.7) and (4.8) in a different form for the following theorem by introducing the variables $u$ and $v$ which are defined such that $\partial_{u}=\partial_{x}-\sigma \partial_{y}$ and $\partial_{v}=\partial_{x}+\sigma \partial_{y}$.

Theorem 4.1. Consider the following system of partial differential equations:

$$
\begin{gathered}
H_{u v}=\frac{1}{2} R H, \\
H_{z}=-\alpha H_{u u u}-\beta H_{v v v}+3 \alpha W_{2 u} H_{u}+3 \beta W_{1 v} H_{v}
\end{gathered}
$$

where $\alpha$ and $\beta$ are constants with $W_{1}$ and $W_{2}$ given by

$$
W_{1}=\frac{1}{2} \int_{-\infty}^{u} R d \tilde{u}=-\frac{1}{2} \partial_{u}^{-1} R, \quad W_{2}=\frac{1}{2} \int_{-\infty}^{v} R d \widetilde{v}=\frac{1}{2} \partial_{v}^{-1} R,
$$

Then the function $R$ satisfies the following partial differential equation:

$$
R_{z}+\alpha R_{u u u}+\beta R_{v v v}-3 \alpha\left(R W_{2 u}\right)_{u}-3 \beta\left(R W_{1 v}\right)_{v}=0, \quad W_{1, u}=W_{2, v}=\frac{1}{2} R .
$$

The proof of this goes along exactly the same lines as the previous theorems. It suffices to substitute the integrals (4.10) into (4.9) and compute the compatibility condition by calculating the derivatives $H_{z u v}, H_{u v z}$, which is some work, and then putting them in the equation $H_{z u v}-H_{u v z}=0$. This generates (4.11).

The result in (4.11) is the simplest and lowest member of a hierarchy of nonlinear partial differential equations which is referred to as the Nizhnik-Veselov-Novikov equation. This equation is integrable by means of the IST method. Its properties are different for the cases $\sigma^{2}= \pm 1$ and $E>0, E<0$ and $E=0$.

Continuing further, some nonlinear partial differential equations will be produced which are associated with the scalar curvature equation (4.3). 
The first example to be considered corresponds to selecting $H_{1}=H_{2}=H$ and $H_{3}=1$, which implies that the metric has the form

$$
d s^{2}=H^{2} d x_{1}^{2}-\sigma^{2} H^{2} d x_{2}^{2}-d x_{3}^{2} .
$$

Equation (4.3) for the scalar curvature $R$ then simplifies to the form

$$
(\ln H)_{x x}-\sigma^{2}(\ln H)_{y y}-2 H H_{z z}-H_{z}^{2}+\frac{1}{2} R H^{2}=0
$$

Suppose, for example, that we take $H=\exp \varphi$ so that upon differentiation $H_{z}=\varphi_{z} e^{\varphi}$ and $H_{z z}=\varphi_{z}^{2} e^{\varphi}+\varphi_{z z} e^{\varphi}$, then the equation takes the form

$$
\varphi_{x x}-\sigma^{2} \varphi_{y y}-\left(2 \varphi_{z z}+3 \varphi_{z}^{2}\right) e^{2 \varphi}+\frac{1}{2} \operatorname{Re}^{2 \varphi}=0
$$

If instead we take $e_{3}=+1$ in the metric, the equation obtained from (4.3) is given by

$$
\varphi_{x x}-\sigma^{2} \varphi_{y y}+\left(2 \varphi_{z z}+3 \varphi_{z}^{2}\right) e^{2 \varphi}+\frac{1}{2} \operatorname{Re}^{2 \varphi}=0
$$

When $\varphi_{z}=0$ and $R$ is put equal to a constant value, (4.15) reduces to the Liouville equation.

Another interesting case is the one in which trigonometric functions appear in the metric. To have an example of this case, take $e_{1}=1, e_{2}=\mp \sigma^{2}$ and $e_{3}=\mp 1$ with $\theta=\theta(x, y, z)$, and $H_{j}$ are given by

$$
H_{1}=\cos \frac{\theta}{2}, \quad H_{2}=\sin \frac{\theta}{2}, \quad H_{3}=1
$$

Equation (4.3) can be simplified to the form

$$
\theta_{x x} \pm \sigma^{2} \theta_{y y} \mp \cos \theta \theta_{z z} \pm \frac{3}{4} \sin \theta \theta_{z}^{2}-\frac{1}{4} R \sin \theta=0 .
$$

Suppose that $e_{1}=1, e_{2}=-\sigma^{2}$, and $e_{3}=-1$ such that

$$
H_{1}=\cos \theta, \quad H_{2}=\sin \theta, \quad H_{3}=\theta_{z} .
$$


The following equation results from (4.3):

$$
\begin{aligned}
& \frac{\sin \theta}{\cos \theta} \theta_{x x z}-\sigma^{2} \frac{\cos \theta}{\sin \theta} \theta_{y y z}+\left(\theta_{x x}+\sigma^{2} \theta_{y y}\right) \theta_{z}+\frac{1}{2 \cos ^{2} \theta}\left(\theta_{x}^{2}\right)_{z}+\frac{\sigma^{2}}{2 \sin ^{2} \theta}\left(\theta_{y}^{2}\right)_{z} \\
& +\left(3-\frac{1}{4} R\right) \sin \theta \cos \theta \theta_{z}=0 .
\end{aligned}
$$

Equation (4.19) can be considered to be a type of generalized sine-Gordon equation.

Another interesting equation results if we take $e_{1}=1, e_{2}=-\sigma^{2}$, and $e_{3}=-1$ with

$$
H_{1}=e^{\varphi}, \quad H_{2}=e^{\varphi}, \quad H_{3}=\varphi_{z} .
$$

The equation for $\varphi$ obtained from (4.3) using (4.20) is given by

$$
\left(\varphi_{x x}-\sigma^{2} \varphi_{y y}\right)_{z}+\left(\varphi_{x x}-\sigma^{2} \varphi_{y y}\right) \varphi_{z}+\left(3+\frac{1}{4} R\right) \varphi_{z} e^{2 \varphi}=0
$$

Integrating this equation with respect to $z$, the following system is obtained:

$$
\varphi_{x x}-\sigma^{2} \varphi_{y y}-\tau e^{-\varphi}+e^{2 \varphi}=0, \quad \tau_{z}=\left(6+\frac{1}{4} R\right) \varphi_{z} e^{3 \varphi} .
$$

To prove (4.22), multiply the first equation by $e^{\varphi}$ and differentiate both sides with respect to $z$ to obtain

$$
\left(\varphi_{x x}-\sigma^{2} \varphi_{y y}\right)_{z} e^{\varphi}+\left(\varphi_{x x}-\sigma^{2} \varphi_{y y}\right) \varphi_{z} e^{\varphi}-\tau_{z}+3 \varphi_{z} e^{3 \varphi}=0
$$

Substituting $\tau_{z}$ from (4.22) and factoring $e^{\varphi}$, the original equation (4.21) is obtained.

For the case in which the scalar curvature $R$ is independent of $z$, the second equation in (4.22) can be written as

$$
\tau_{z}=\frac{1}{3}\left(6+\frac{1}{4} R\right)\left(e^{3 \varphi}\right)_{z}
$$

By integration, this implies that $\tau$ is given by

$$
\tau=\left(2+\frac{1}{12} R\right) e^{3 \varphi}+\xi(x, y)
$$

where $\xi(x, y)$ is an arbitrary function of $(x, y)$. Substituting $\tau$ from (4.25) into the first equation of (4.22), we get

$$
\varphi_{x x}-\sigma^{2} \varphi_{y y}-\xi(x, y) e^{-\varphi}-\left(1+\frac{R}{12}\right) e^{2 \varphi}=0 .
$$


Another interesting case to examine is the one in which the metric is given by $d s^{2}=H^{2}\left(d x^{2}+\right.$ $\left.d y^{2}+d z^{2}\right)$. For the metric in this form, (4.3) reduces to

$$
H_{x x}+H_{y y}+H_{z z}-\frac{1}{2 H}\left(H_{x}^{2}+H_{y}^{2}+H_{z}^{2}\right)-\frac{1}{8} R H^{3}=0 .
$$

In terms of a function $\varphi$ which is defined by $H=\varphi^{2}$, this equation reduces to the following form:

$$
\varphi_{x x}+\varphi_{y y}+\varphi_{z z}-\frac{R}{16} \varphi^{5}=0 .
$$

Finally, some equations relevant to a four-dimensional Riemann space with diagonal metric which is an extension of (4.1) will be determined. The metric is written as

$$
d s^{2}=e_{1} H_{1}^{2} d x_{1}^{2}+e_{2} H_{2}^{2} d x_{2}^{2}+e_{3} H_{3}^{2} d x_{3}^{2}+e_{4} H_{4}^{2} d x_{4}^{2}
$$

where $e_{i}= \pm 1$ as before, and $H_{j}$ are functions to be specified in a given case. The scalar curvature can be calculated by means of (4.2) given the components of metric (4.29). Some equations will be determined based on metric (4.29) in the following.

(i) Suppose that we take $e_{1}=1, e_{2}=\sigma^{2}, e_{3}=1$, and $e_{4}=\sigma^{2}$ with the functions $H_{1}=H_{2}=H_{3}=\exp (\varphi), H_{4}=1$. Equation (4.2) produces the following equation:

$$
\varphi_{x x}+\varphi_{y y}+\varphi_{z z}+\frac{3}{2} \sigma^{2} e^{2 \varphi} \varphi_{w w}+\frac{1}{2}\left(\varphi_{x}^{2}+\sigma^{2} \varphi_{y}^{2}+\varphi_{z}^{2}+6 \sigma^{2} e^{2 \varphi} \varphi_{w}^{2}\right)-\frac{1}{8} \operatorname{Re}^{2 \varphi}=0 .
$$

(ii) The case in which $e_{1}=1, e_{2}=\sigma^{2}, e_{3}=1$, and $e_{4}=\sigma^{2}$ with functions $H_{1}=H_{2}=e^{\varphi}$ and $H_{3}=H_{4}=1$ yields

$$
\varphi_{x x}+\sigma^{2} \varphi_{y y}+2 e^{2 \varphi} \varphi_{z z}+2 \sigma^{2} e^{2 \varphi} \varphi_{w w}+3 e^{2 \varphi} \varphi_{z}^{2}+3 e^{2 \varphi} \varphi_{w}^{2}-\frac{1}{4} \operatorname{Re}^{2 \varphi}=0 .
$$

(iii) The selection $e_{i}=1$ with $H_{1}=H_{2}=H_{3}=e^{\varphi}$ and $H_{4}=\varphi_{w}$ produces the following equation:

$$
\begin{aligned}
& \frac{1}{2 \varphi_{w}}\left(\varphi_{x x w}+\varphi_{y y w}+\varphi_{z z w}\right) \varphi_{x x}+\varphi_{y y}+\varphi_{z z}+\frac{1}{2}\left(\varphi_{x}^{2}+\varphi_{y}^{2}\right) \\
& \quad+\frac{1}{2 \varphi_{w}}\left(\varphi_{x} \varphi_{x w}+\varphi_{y} \varphi_{y w}+\varphi_{z} \varphi_{z w}\right)+\left(3-\frac{1}{8} R\right) e^{2 \varphi}=0 .
\end{aligned}
$$

(iv) When the metric is diagonal of the form $d s^{2}=H^{2}\left(d x^{2}+d y^{2}+d z^{2}+d w^{2}\right)$ and all $e_{i}=1$, the equation generated by (4.3) is given by

$$
H_{x x}+H_{y y}+H_{z z}+H_{w w}-\frac{1}{24} R H^{3}=0 .
$$


If we take $H=\varphi^{2}$ in (4.33), we obtain the equation

$$
\varphi_{x x}+\varphi_{y y}+\varphi_{z z}+\varphi_{w w}+\frac{1}{\varphi}\left(\varphi_{x}^{2}+\varphi_{y}^{2}+\varphi_{z}^{2}+\varphi_{w}^{2}\right)-\frac{1}{24} R \varphi^{5}=0
$$

(v) As a final example, suppose that $e_{1}=e_{3}=1, e_{2}=e_{4}=-1$ and trigonometric functions are used for the $H_{j}$ in the metric, $H_{1}=H_{3}=\cos \theta, H_{2}=H_{4}=\sin \theta$, then the equation for $\theta$ can be put in the form

$$
\begin{aligned}
& \sin ^{3} \theta \cos \theta\left(\sin ^{2} \theta-2 \cos ^{2} \theta\right)\left(\theta_{x x}+\theta_{z z}\right)+\cos ^{3} \theta \sin \theta\left(\cos ^{2} \theta-2 \sin ^{2} \theta\right)\left(\theta_{y y}+\theta_{w w}\right) \\
& \quad+\left(4 \sin ^{6} \theta-\cos ^{6} \theta\right)\left(\theta_{x}^{2}+\theta_{z}^{2}\right)-\left(4 \cos ^{6} \theta-\sin ^{6} \theta\right)\left(\theta_{y}^{2}+\theta_{w}^{2}\right)+\frac{1}{4} R \sin ^{4} \theta \cos ^{4} \theta=0 .
\end{aligned}
$$

\section{Conclusions}

It has been shown that several equations of physical interest arise within the analysis of the geometry of surfaces, such as the Liouville and Schrödinger equations, or as a result of the compatibility condition of two linear equations. Moreover, these equations can be combined in such a way as to produce integrable evolutions or deformations of surfaces. An introduction to a more systematic approach to going to higher dimensional partial differential equations has been mentioned. Finally, by calculating the scalar curvature based on the Riemannian metric for three- and four-dimensional Riemann spaces, a large group of nonlinear partial differential equations has been determined in both three and four dimensions. It remains as a separate task to begin to study the various kinds of solutions and their characteristics for some of these equations.

\section{References}

[1] D. G. Gross, C. N. Pope, and S. Weinberg, Two Dimensional Quantum Gravity and Random Surfaces, World Scientific, Singapore, 1992.

[2] D. Nelson, T. Piran, and S. Weinberg, Statistical Mechanics of Membranes and Surfaces, World Scientific, Singapore, 1992.

[3] P. Bracken and A. M. Grundland, "Symmetry properties and explicit solutions of the generalized Weierstrass system," Journal of Mathematical Physics, vol. 42, no. 3, pp. 1250-1282, 2001.

[4] P. Bracken, "Partial differential equations which admit integrable systems," International Journal of Pure and Applied Mathematics, vol. 43, no. 3, pp. 409-421, 2008.

[5] M. J. Ablowitz and P. A. Clarkson, Solitons, Nonlinear Evolution Equations and Inverse Scattering, vol. 149 of London Mathematical Society Lecture Note Series, Cambridge University Press, Cambridge, UK, 1991.

[6] P. Bracken, A. M. Grundland, and L. Martina, "The Weierstrass-Enneper system for constant mean curvature surfaces and the completely integrable sigma model," Journal of Mathematical Physics, vol. 40, no. 7, pp. 3379-3403, 1999.

[7] S. S. Chern and K. Tenenblat, "Pseudospherical surfaces and evolution equations," Studies in Applied Mathematics, vol. 74, no. 1, pp. 55-83, 1986.

[8] R. Sasaki, "Soliton equations and pseudospherical surfaces," Nuclear Physics B, vol. 154, no. 2, pp. 343-357, 1979.

[9] F. Calogero and A. Degasperis, Spectral Transform and Solitons. Vol. I, vol. 13 of Studies in Mathematics and Its Applications, North-Holland, Amsterdam, The Netherlands, 1982. 
[10] B. G. Konopelchenko, "Soliton curvatures of surfaces and spaces," Journal of Mathematical Physics, vol. 38, no. 1, pp. 434-457, 1997.

[11] B. G. Konopelchenko, Introduction to Multidimensional Integrable Equations, Plenum, New York, NY, USA, 1992.

[12] S. S. Chern, W. H. Chen, and K. S. Lam, Lectures on Differential Geometry, vol. 1 of Series on University Mathematics, World Scientific, Singapore, 1999.

[13] L. D. Landau and E. M. Lifshitz, Quantum Mechanics, Pergamon Press, Oxford, UK, 1997.

[14] R. Beutler and B. G. Konopelchenko, "Surfaces of revolution via the Schrödinger equation: construction, integrable dynamics and visualization," Applied Mathematics and Computation, vol. 101, no. 1, pp. 13-43, 1999.

[15] P. Bracken, "Dynamics of induced surfaces in four-dimensional Euclidean space," Pacific Journal of Applied Mathematics, vol. 1, no. 2, pp. 207-220, 2008.

[16] I. A. Taimanov, "Surfaces of revolution in terms of solitons," Annals of Global Analysis and Geometry, vol. 15, no. 5, pp. 419-435, 1997.

[17] B. G. Konopelchenko, "Induced surfaces and their integrable dynamics," Studies in Applied Mathematics, vol. 96, no. 1, pp. 9-51, 1996.

[18] B. W. Char, K. O. Geddes, B. L. Leong, M. Monagen, and S. Watt, Maple V Language Reference Manual, Springer, New York, NY, USA, 1991.

[19] V. E. Zakharov, "Integrable systems in multidimensional spaces," in Mathematical Problems in Theoretical Physics, vol. 153 of Lecture Notes in Physics, pp. 190-216, Springer, Berlin, Germany, 1982. 


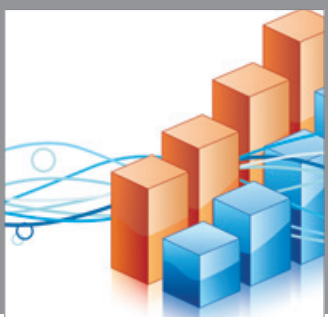

Advances in

Operations Research

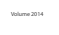

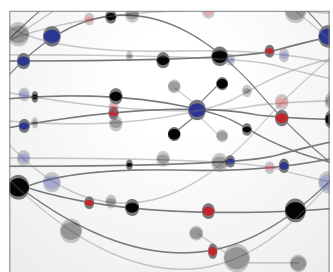

\section{The Scientific} World Journal
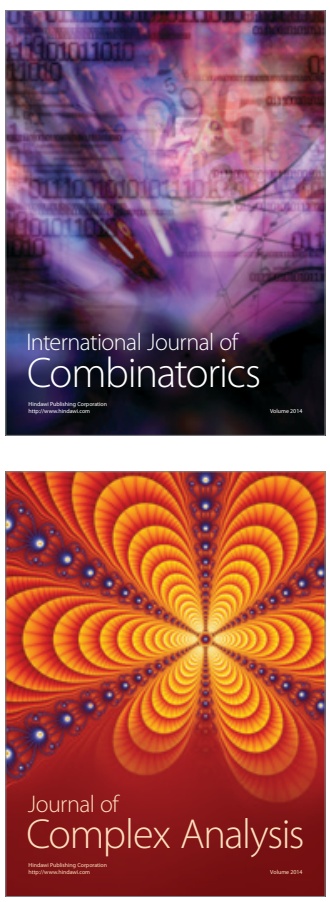

International Journal of

Mathematics and

Mathematical

Sciences
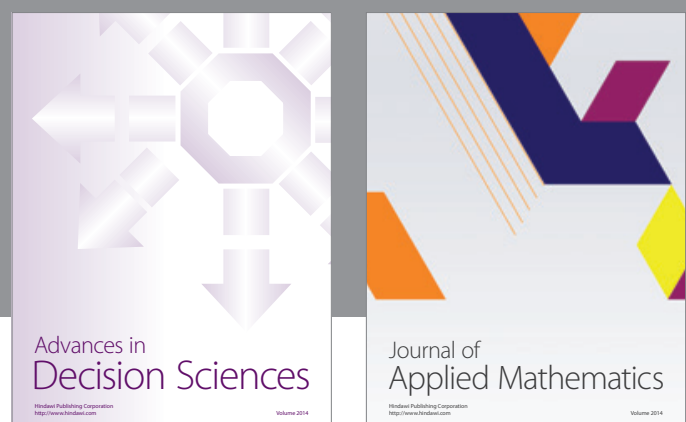

Journal of

Applied Mathematics
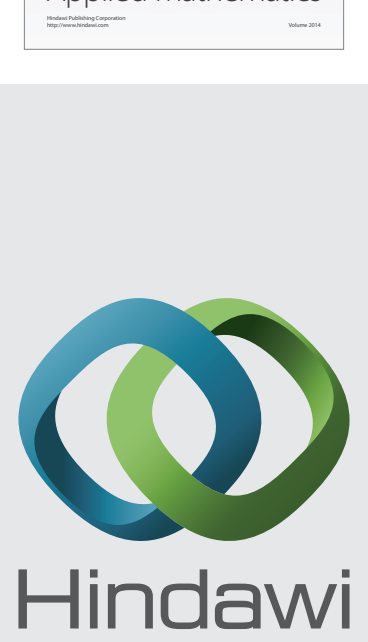

Submit your manuscripts at http://www.hindawi.com
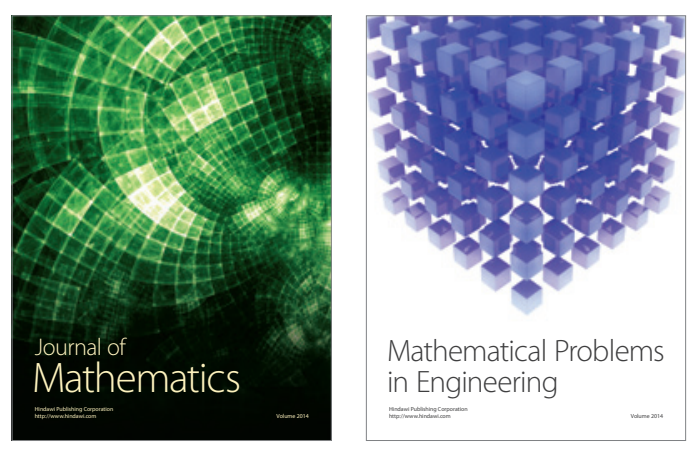

Mathematical Problems in Engineering
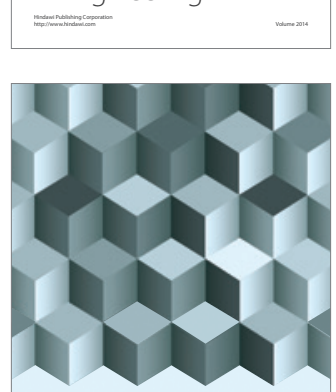

Journal of

Function Spaces
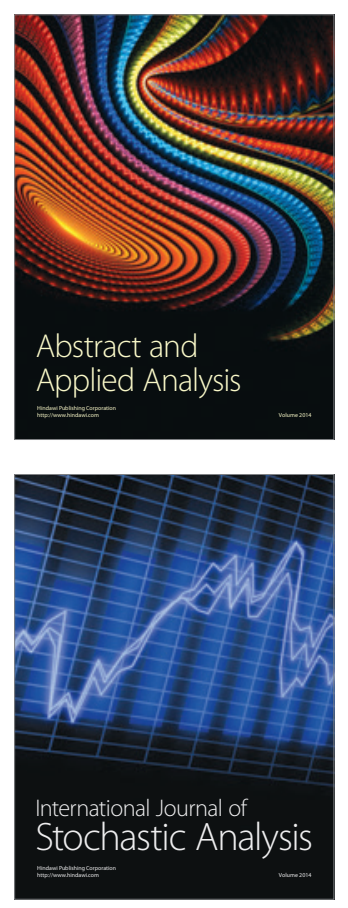

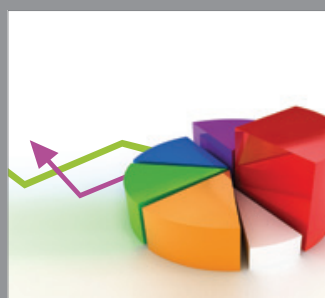

ournal of

Probability and Statistics

Promensencen
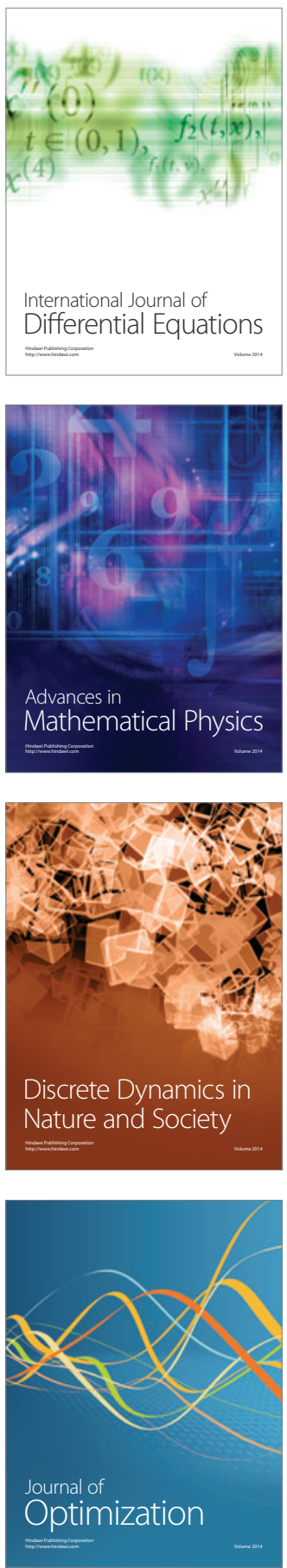